Pacific Journal of Mathematics

EQUALLY PARTITIONED GROUPS 


\section{EQUALLY PARTITIONED GROUPS}

I. M. ISAACS

It is proved that the only finite groups which can be partitioned by subgroups of equal orders are the $p$-groups of exponent $p$. The connection between equally partitioned groups and Sperner spaces is discussed. It is also proved that finite groups partitioned by pairwise permutable subgroups are abelian.

1. Let $G$ be a group and let $\Pi$ be a collection of proper subgroups of $G$. Then $I$ is said to partition $G$ if every nonidentity element of $G$ is contained in exactly one $H \in \Pi$. If $G$ is a $p$-group of exponent $p$ and $|G|>p$, we may let $\Pi$ be the set of cyclic subgroups of $G$. Then $\Pi$ is a partition consisting of subgroups of equal finite orders. Our main result is that the $p$-groups of exponent $p$ are the only finite groups which can be equally partitioned.

The methods of proof in this paper depend strongly on the finiteness of the group and give no information about which infinite groups can be partitioned by subgroups of equal finite orders.

I began to consider equally partitioned groups after attending a lecture by Prof. A. Barlotti on Sperner spaces. Examples of these geometric objects (which generalize affine spaces) are provided by such groups. In fact the Sperner spaces which arise from finite equally partitioned groups are exactly those which Barlotti and Cofman [2] call translation spaces. This will be discussed further in $\S 3$.

2. Only finite groups will be considered. A great deal is known about partitioned groups. (We mention in particular the papers [1] and [5].) Our theorem, however, is much more elementary and does not depend on the deeper results.

The following easy lemma (which appears in [1]) is crucial to the study of partitioned groups.

Lemma 1. Let $G$ be partitioned by $\Pi$ and let $x, y \in G-\{1\}$ with $x y=y x$. Suppose $x$ and $y$ lie in different elements of $\Pi$. Then $x$ and $y$ have equal prime orders.

Proof. Suppose $o(x)<o(y)$. Then $(x y)^{o(x)}=y^{o(x)} \neq 1$. Let $y \in H$ $\in \Pi$ then $(x y)^{\circ(x)} \in H$ and hence $x y \in H$. Thus $x \in H$, a contradiction. Therefore $o(x)=o(y)$. Similarly, $o\left(x^{n}\right)=o(y)=o(x)$ for positive integers $n<o(x)$. It follows that $o(x)$ is prime.

LEMma 2. Let $G$ be equally partitioned by $\Pi$ and let $X \leqq G$ be 
a subset, $X \nsubseteq\{1\}$. Then there exists $H \in \Pi$ such that $H$ contains no conjugate of $X$.

Proof. Suppose that the lemma is false and for each $H \in \Pi$, choose $X_{H}$ conjugate to $X$ with $X_{H} \subseteq H$. Let $N_{H}=N_{H}\left(X_{H}\right)$ so that $H$ contains at least $\left|H: N_{H}\right|$ conjugates of $X$. Let $N=N_{G}(X)$. Then if $|G|=g$ and $|H|=h$ for $H \in \Pi$, we have

$$
|G: N|=\left|G: N_{G}\left(X_{H}\right)\right| \leqq\left|G: N_{H}\right|=|G: H|\left|H: N_{H}\right|
$$

and hence

$$
\left|H: N_{I I}\right| \geqq h|G: N| / g \text {. }
$$

Now $|G: N|$ is the number of conjugates of $X$ in $G$ and thus

$$
\begin{aligned}
|G: N| & \geqq \sum_{H \in \Pi}\left|H: N_{I I}\right| \\
& \geqq|\Pi||G: N| h / g .
\end{aligned}
$$

However, $|\Pi|=(g-1) /(h-1)>g / h$ and this yields a contradiction.

Note. It follows from Lemma 2 that if $G$ is equally partitioned by $\Pi$, then no element of $\Pi$ can contain a full Sylow $p$-subgroup of $G$ for any $p|| G \mid$. Otherwise, every $H \in \Pi$ would contain an $S_{p}$ subgroup, violating the lemma.

Lemma 3. Let $G$ be equally partitioned. Then every element of $G$ has prime order.

Proof. Suppose that $x \in G$ has composite order and let $\mathscr{K}$ be the conjugacy class of $x$. Let $\Pi$ be the given partition. By Lemma 2, there exists $H \in \Pi$ with $H \cap \mathscr{C}=\dot{\phi}$. By Lemma 1, no element of $H$ centralizes any element of $\mathscr{K}$. Thus $H$ acts semi-regularly on $\mathscr{K}$ and hence $|H||| \mathscr{C} \mid$.

Now pick $K \in \Pi$ with $x \in K$. Then $K$ acts semi-regularly by conjugation on $\mathscr{K}-K$ so that $|K|||(\mathscr{K}-K) \mid$. Since $|H|=|K|$, we conclude that $|K||| \mathscr{K} \cap K \mid$. This is a contradiction because $0<|\mathscr{C} \cap K|<|K|$.

The next two results are routine applications of standard facts. We include them for completeness.

LemMA 4. Suppose $G$ has a nontrivial normal p-subgroup where $p$ is the largest prime divisor of $|G|$. Assume that every element of $G$ has prime order and let $P \in \operatorname{Syl}_{p}(G)$. Then either $P=G$ or $|G: P|$ is prime and $P \triangleleft G$. 
Proof. Let $1 \neq U \triangleleft G$ where $U$ is a $p$-group. Now $G$ can contain no subgroup, $W$, of order $q r$ where $q$ and $r$ are (possibly equal) primes different from $p$. This is so since otherwise $C_{U}(w)=1$ for all $1 \neq w \in W$ and this forces $W$ to be cyclic (Satz V. 8. 15b of [3]).

There is nothing to prove if $P=G$ so suppose $P<G$ and let $q$ be the smallest prime divisor of $|G|$. Let $Q \in \operatorname{Syl}_{p}(G)$. Then $|Q|=q$ and thus $G$ has a normal $q$-complement, $M$.

If $M=P$, the proof is complete. Suppose that $P<M$. Then $Q$ normalizes some $R \in \operatorname{Syl}_{r}(M)$ for $r \neq p$. Thus $|R|=r$ and $|Q R|=q r$, a contradiction.

Corollary 5. Assume that every element of $G$ has prime order. Let $P \in \operatorname{Syl}_{p}(G)$, where $p$ is the largest prime divisor of $|G|$. Then $P$ is a T. I. set (i.e., $P \cap P^{x}=1$ for all $x \notin N(P)$ ).

Proof. Assume that the corollary is false and let $1<D=P \cap P^{x}$ where $P \neq P^{x}$ and $|D|$ is maximal. Then $N_{G}(D)=N$ does not have a unique Sylow $p$-subgroup. This violates Lemma 4 as applied to $N$.

Theorem 6. Let $G$ be equally partitioned. Then $G$ is a p-group of exponent $p$.

Proof. Let $p$ be the largest prime divisor of $|G|$ and let $P \in \operatorname{Syl}_{p}(G)$. By Lemmas 3 and $4, N(P)=P C$ where either $C=1$ or $|C|=q$, a prime. By Corollary 5, $P$ is a T. I. set.

We establish some notation. Let $|G|=g,|P|=p^{b}$ and $|C|=c$. Let $\Pi$ be the given partition and let $|H|=h$ for all $H \in \Pi$. Let $p^{a}$ be the $p$-part of $h$.

Since $P$ is a T. I. set, it follows that $P \cap U \in \operatorname{Syl}_{p}(U)$ for all subgroups $U \subseteq G$ with $P \cap U \neq 1$. Thus $|P \cap H|=p^{a}$ for all $H \in \Pi$ such that $P \cap H \neq 1$. Since $P=\bigcup_{H \in I}(P \cap H)$, it follows that $\left(p^{a}-1\right) \mid\left(p^{b}-1\right)$. We can also conclude from the fact that $P$ is a T. I. set that $G$ contains exactly $g\left(p^{b}-1\right) / p^{b} c$ elements of order $p$.

Now by Lemma 2, we may choose $H \in \Pi$ with $H \cap C^{g}=1$ for all $g \in G$. Let $P_{0} \in \operatorname{Syl}_{p}(H)$. We may assume that $P_{0} \subseteq P$. Since $P$ is a T. I. set, $N_{H}\left(P_{0}\right) \subseteq N_{G}(P)=P C$. It follows that $N_{H}\left(P_{0}\right)=P_{0} C_{0}$ where $C_{0} \subseteq C^{g}$ for some $g$. Thus $C_{0}=1$ and $P_{0}=N_{H}\left(P_{0}\right)$. By Sylow's Theorem it follows that $h / p^{a} \equiv 1 \bmod p$.

Let $K \in \Pi$ and let $P_{1} \in \operatorname{Syl}_{p}(K)$. Reasoning as above, we conclude that $N_{K}\left(P_{1}\right)=P_{1} C_{1}$ where $C_{1} \subseteq C^{x}$ for some $x$. Thus $h /\left(p^{a}\left|C_{1}\right|\right) \equiv 1$ $\bmod p$ and hence $\left|C_{1}\right| \equiv 1 \bmod p$. However, $\left|C_{1}\right|=1$ or $q$ where $q$ is a prime $<p$. It follows that $C_{1}=1$ and thus every $K \in \Pi$ has selfnormalizing Sylow $p$-subgroups.

Since the Sylow $p$-pubgroups of $K \in \Pi$ are T. I. sets, it follows 
that each such $K$ contains exactly $h\left(p^{a}-1\right) / p^{a}$ elements of order $p$. Since $|\Pi|=(g-1) /(h-1)$, this yields

$$
g\left(p^{b}-1\right) / p^{b} c=(g-1) h\left(p^{a}-1\right) /(h-1) p^{a} .
$$

Since $g / h<(g-1) /(h-1)$, we conclude from (1) that

$$
1 / c>\left(p^{b}-1\right) / p^{b} c>\left(p^{a}-1\right) / p^{a}=1-1 / p^{a} \geqq 1 / 2
$$

and thus $c=1$. Now (1) yields

$$
(g-1) h\left(p^{a}-1\right) p^{b}=(h-1) g\left(p^{b}-1\right) p^{a} .
$$

Since $\left((g-1), g p^{a}\right)=1$ and $\left(p^{b}-1\right) /\left(p^{a}-1\right)$ is an integer, we obtain

$$
g p^{a} \mid h p^{b} \text {. }
$$

The $p$-parts of $g p^{a}$ and $h p^{b}$ are equal and $h \mid g$. It follows that $h p^{b} \mid g p^{a}$ and thus

$$
h p^{b}=g p^{a} .
$$

Combining this with (2) yields

$$
(h-1)\left(p^{b}-1\right)=(g-1)\left(p^{a}-1\right)
$$

and subtracting (4) from (3), one obtains

$$
h+p^{b}=g+p^{a} \text {. }
$$

Since $h \mid g$ and $h<g$, we have

$$
g / 2 \leqq g-h=p^{b}-p^{a}<p^{b} .
$$

Since $p^{b} \mid g$, we conclude that $p^{b}=g$ and the result follows.

Note. Once it was established that $c=1$, above, the proof could have been finished using Frobenius' Theorem, ([3], Hauptsatz V. 7. 6). Since $P$ is a self-normalizing T. I. set, Frobenius' Theorem yields a normal $p$-complement, $U$, for $G$. Also $C_{U}(x)=1$ for all $1 \neq x \in P$. If $U \neq 1$, it follows from the fact that $P$ has exponent $p$ that $|P|=p$. A contradiction now results by applying the note following Lemma 2 .

3. In this section we discuss the connection between Sperner spaces and equally partitioned groups.

Definition. ([4].) A Sperner space is a set, $S$, of "points" and a collection, $\mathscr{L}$, of proper finite subsets of $S$, called "lines" such that

(a) every two points determine a unique line,

(b) all lines have equal numbers of points, 
(c) an equivalence relation (called "parallelism") is defined on $\mathscr{L}$ and

(d) for each $x \in S$, there is exactly one line which contains $x$ in each parallel class.

If $G$ is a group which is equally partitioned by $\Pi$, we may define a Sperner space by taking $S=G, \mathscr{L}=\{H x \mid H \in \Pi, x \in G\}$ and setting $(H x) \|(K y)$ if and only if $H=K$. It is routine to check that this does define a Sperner space. We denote this space by $S(G, \Pi)$.

Given a Sperner space, $(S, \mathscr{L})$, we consider the groups, $G(S, \mathscr{L})$, consisting of all those collineations of $S$ which map each line to a line parallel to itself. Since no two distinct parallel lines of $(S, \mathscr{L})$ can intersect (by condition (d)), it follows that if $g \in G(S, \mathscr{L}$ ) fixes a point, $x \in S$, then $g$ fixes every line through $x$. It now follows easily that only the identity of $G(S, \mathscr{L})$ fixes two points of $S$.

Let $G_{0}(S, \mathscr{L})=\{1\} \cup\{g \in G(S, \mathscr{L}) \mid g$ fixes no points of $S\}$. In [2], Barlotti and Cofman call a Sperner space $(S, \mathscr{L})$ a translation space if $G_{0}(S, \mathscr{L})$ is a group which is transitive on $S$. If $S$ is finite, it follows from Frobenius' Theorem ([3], Satz. V. 8. 2 (a)) that $(S, \mathscr{L})$ is a translation space if and only if $G(S, \mathscr{L})$ is transitive on $S$. If $(G, \Pi)$ is a finite equally partitioned group and $(S, \mathscr{L})=S(G, \Pi)$, then $G(S, \mathscr{L})$ contains right multiplications by elements of $G$ and hence is transitive. It follows that $S(G, \Pi)$ is a translation space and $G_{0}(S, \mathscr{L})$ is the group of right multiplications.

We claim that if $(S, \mathscr{L})$ is any finite translation space then $(S, \mathscr{L}) \cong S(G, \Pi)$ for some equally partitioned group $(G, \Pi)$. Let $G=G_{0}(S, \mathscr{L})$ and choose a point $e \in S$. For $l \in \mathscr{L}$, let $H_{l}$ be the (setwise) stabilizer of $l$ in $G$ and let $\Pi=\left\{H_{l} \mid l \in \mathscr{L}\right.$ and $\left.e \in l\right\}$. If $e, x \in l$ and $g \in G$ with $e g=x$, then $x \in l \cap l g$ and thus $l=l g$ and $g \in H_{l}$. It follows that $H_{l}$ is transitive on $l$ and $\left|H_{l}\right|=|l|$. Therefore, all $H \in \Pi$ have equal order. If $H, K \in \Pi$ with $H \neq K$, then $H \cap K$ fixes $e$ and hence $H \cap K=1$. Also

$$
\begin{aligned}
|G|=|S| & =1+\Sigma\{|l|-1 \mid l \in \mathscr{L} \text { and } e \in l\} \\
& =1+\Sigma\{|H|-1 \mid H \in \Pi\}=|\bigcup \Pi \Pi|
\end{aligned}
$$

and thus $\Pi$ is a partition for $G$.

To see that $S(G, \Pi) \cong(S, \mathscr{L})$, define $\theta: G \rightarrow S$ by $\theta(g)=e g$. It is routine to show that $\theta$ is an isomorphism of Sperner spaces.

One further remark on the correspondence between finite translation spaces and finite equally partitioned groups is in order. If $(G, \Pi)$ and $\left(G_{1}, \Pi_{1}\right)$ are two equally partitioned groups such that $S(G, \Pi) \cong$ $S\left(G_{1}, \Pi_{1}\right)$, then $G \cong G_{1}$ and this group isomorphism can be chosen so as to carry $\Pi$ to $\Pi_{1}$. This follows since $G \cong G_{0}(S(G, \Pi))$ and under this (natural) isomorphism, $\Pi$ corresponds exactly to the set of 
stabilizers of the lines through 1.

Let $(S, \mathscr{L})$ be a finite translation space. By Theorem $6,|S|=p^{b}$ for some prime, $p$, and $|l|=p^{a}$ for $l \in \mathscr{L}$. Also, $\left(p^{a}-1\right) \mid\left(p^{b}-1\right)$ and as is well known, this forces $a \mid b$. We may define the dimension of $(S, \mathscr{L})$ to be $b / a$.

Let $q=p^{a}$ and let $K=\mathrm{GF}(q)$. Let $V$ be a vectorspace of dimension $n$ over $K$ and let $\Pi$ be the set of one-dimensional subspaces of $V$. Then $\Pi$ equally partitions $V$ and of course $S(V, \Pi)$ is an affine space of dimension $n$. This suggests the question of which translation spaces, $(S, \mathscr{L})$, correspond to abelian equally partitioned groups. These are not necessarily affine although they do satisfy the following condition:

$\left(^{*}\right) \quad$ Let $l, m \in \mathscr{L}$ with $l \cap m \neq \varnothing$. Let $x \in l$ and $y \in m$. Let $l^{\prime} \| l$ with $y \in l^{\prime}$ and $m^{\prime} \| m$ with $x \in m^{\prime}$. Then $l^{\prime} \cap m^{\prime} \neq \varnothing$.

It is easy to see that $S(G, \Pi)$ satisfies $\left(^{*}\right)$ if and only if for every $H, K \in \Pi$ and every $h \in H$ and $k \in K$ we have $H k \cap K h \neq \varnothing$. This condition is clearly satisfied if $G$ is abelian since then $h k \in H k \cap K h$. In the next section we prove that only in abelian groups does this condition hold.

4. We begin with the following lemma.

Lemma 7. Let $H, K \leqq G$. Then $H K=K H$ if and only if for every $h \in H$ and $k \in K$ we have $H k \cap K h \neq 1$.

Proof. Suppose $H K=K H$. Let $h \in H$ and $k \in K$. Then $k h^{-1} \in$ $K H=H K$ and $k h^{-1}=h_{1}^{-1} k_{1}$ for some $h_{1} \in H$ and $k_{1} \in K$. Thus $h_{1} k=$ $k_{1} h \in H k \cap K h$.

Conversely, let $x \in K H$. Write $x=k h^{-1}$ for some $k \in K$ and $h \in H$. Now choose $k_{1} h=h_{1} k \in K h \cap H k$ so that $k_{1} \in K$ and $h_{1} \in H$. Then $x=k h^{-1}=h_{1}^{-1} k_{1} \in H K$ and $K H \subseteq H K$. The reverse inclusion follows symmetrically and the proof is complete.

The main result of this section is the following.

Theorem 8. Let $G$ be a finite group partitioned by II. Assume that $H K=K H$ for all $H, K \in \Pi$. Then $G$ is an elementary abelian p-group.

Note that we do not assume that all elements of $I I$ have equal order. Theorem 8 and Lemma 7 prove the claim made at the end of $\S 3$. To prove Theorem 8 , we strengthen it somewhat and use induction.

Theorem 9. Let $G$ be finite and partitioned by II. Suppose $A \in \Pi$ and $A H=H A$ for all $H \in \Pi$. Then $A \triangleleft G$. 
Proof. We use induction on $|G|$. If $A<L<G$, then $L$ is partitioned by $\Pi_{0}=\{H \cap L \mid H \in \Pi\}$. If $H \in \Pi$, then $A H$ is a group and $A H \cap L=A(H \cap L)$. Thus $A(H \cap L)=(H \cap L) A$ and by induction $A \triangleleft L$. Let $N=N(A)$. If $H \in \Pi$ and $A H<G$, it follows that $A \triangleleft A H$ and $H \subseteq N$.

Assume $N<G$ and let $\Pi_{1}=\{H \in \Pi \mid H \nsubseteq N\}$. Then $H A=G$ for all $H \in \Pi_{1}$ and hence $|H|=|G: A|$ for these $H$. Also for $H \in \Pi_{1}$, we have $N=A(N \cap H)$ and thus $|N \cap H|=|N: A|$.

Now

$$
G-N=\bigcup\left\{H-(H \cap N) \mid H \in \Pi_{1}\right\}
$$

and since this union is disjoint, we obtain

$$
|G|-|N|=\left|\Pi_{1}\right|(|G: A|-|N: A|) \text {. }
$$

Solving this yields $\left|\Pi_{1}\right|=|A|$.

Now

$$
\begin{aligned}
\left|\cup \Pi_{1}\right| & =1+\left|\Pi_{1}\right|(|G: A|-1) \\
& =1+|G|-|A| .
\end{aligned}
$$

It follows that $\Pi=\Pi_{1} \cup\{A\}$ and every element of $G-A$ lies in some $H \in \Pi_{1}$.

Let $g \in G$. To show that $A^{g}=A$, it suffices to show that $A^{g} \cap H=1$ for all $H \in \Pi_{1}$. Choose $H \in \Pi_{1}$. Since $G=A H$, we may write $g=a h$ for some $a \in A$ and $h \in H$. Then

$$
A^{g} \cap H=A^{h} \cap H=(A \cap H)^{h}=1
$$

and the proof is complete.

Proof of Theorem 8. By Theorem 9 we have $H \triangleleft G$ for all $H \in \Pi$. Therefore, if $H, K \in \Pi, H \neq K$ we have $K \subseteq C(H)$ and hence $G=H \cup C(H)$. Since $H<G$, we have $G=C(H)$ and $H \leqq Z(G)$. It follows that $G$ is abelian. The result now follows by Lemma 1 .

5. In this section we discuss a class of examples of equally partitioned groups. Since every $p$-group of exponent $p$ is equally partitioned by its cyclic subgroups, it is interesting to look for examples of groups partitioned by subgroups of order $q=p^{a}>p$. The elementary abelian groups of order $q^{n}$ have this property. Nonabelian examples are provided by the next result if $p>2$.

Theorem 10. Let $n \leqq p$ and $q=p^{e}$. Then the Sylow p-subgroups of $\mathrm{GL}(n, q)$ are partitioned by abelian subgroups of order $q$. 
Note. If $n>p$, then the Sylow $p$-subgroups of $\operatorname{GL}(n, q)$ do not have exponent $p$ and hence cannot be equally partitioned.

Proof of Theorem 10. Let $K=G F(q)$ and let $A$ be the space of strictly upper triangular $n \times n$ matrices over $K$. Then $P=\{I+a \mid a \in A\}$ is a Sylow $p$-subgroup of $\operatorname{GL}(n, q)$. For $a \in A$, let $M_{a}(t)=\exp (a t)$ for $t \in K$. This is well defined since $(a t)^{n}=0$ and $n \leqq p$. Since $M_{a}(s) M_{a}(t)=M_{a}(s+t)$, we conclude that $P_{a}=\left\{M_{a}(t) \mid t \in K\right\}$ in an abelian subgroup of $P$.

We will show that if $a, b \in A$ and $\exp (a)=\exp (b)$, then $a=b$. It will follow that $\left|P_{a}\right|=q$ if $a \neq 0$ and that $P_{a} \cap P_{b}=1$ unless $b=a t$ for some $t \in K$; in which case $P_{a}=P_{b}$. Taking $\Pi=\left\{P_{a} \mid 0 \neq a \in A\right\}$ we have $|\Pi|=(|A|-1) /(q-1)$ and

$$
|\bigcup \Pi|=|\Pi|(q-1)+1=|A|=|P|
$$

as desired.

Suppose then that $\exp (a)=\exp (b)$. For $m \in Z, \exp (m a)=\exp (a)^{m}$ and thus $\exp (a t)=\exp (b t)$ for all $t \in \mathrm{GF}(p)$. Let $x$ be an indeterminate and let $E(x)=\exp (a x)-\exp (b x)$. Then $E(x)$ is a matrix with polynomial entries of degree $<p$. Since $E(t)=0$ for all $t \in \operatorname{GF}(p)$, it follows that $E(x)$ is identically 0 . Comparing coefficients of $x$ yields $a=b$ and the proof is complete.

We close with the following question: Does there exist a group partitioned by subgroups of equal order not all of which are abelian?

\section{REFERENCES}

1. R. Baer, Partitionen endicher Gruppen, Math. Zeit., 75 (1961), 333-372.

2. A. Barlotti and J. Cofman, Finite Sperner spaces constructed from projective and affine spaces, to appear.

3. B. Huppert, Endliche Gruppen I, Springer-Verlag, Berlin, 1967.

4. E. Sperner, Affine Raume mit schwacher Inzidenz und zugehörige algebraische Structuren, J. Reine Angew. Math., 204 (1960), 205-215.

5. M. Suzuki, On a finite group with a partition, Arch. Math., 12 (1961), 241-254.

Received July 20, 1972. Research partially supported by NSF grant GP-32813X. 


\section{PACIFIC JOURNAL OF MATHEMATICS}

\section{EDITORS}

RICHARD ARENS (Managing Editor) University of California

Los Angeles, California 90024

R. A. Beaumon'T

University of Washington Seattle, Washington 98105
J. Dugundj1*

Department of Mathematics University of Southern California Los Angeles, California 90007

D. Gilbarg and J. Milgram Stanford University Stanford, California 94305

\section{ASSOCIATE EDITORS}

E. F. BECKENBACH

B. H. NeUMANN

F. WOLF

K. YOSHIDA

\section{SUPPORTING INSTITUTIONS}

UNIVERSITY OF BRITISH COLUMBIA CALIFORNIA INSTITUTE OF TECHNOLOGY UNIVERSITY OF CALIFORNIA MONTANA STATE UNIVERSITY UNIVERSITY OF NEVADA NEW MEXICO STATE UNIVERSITY OREGON STATE UNIVERSITY UNIVERSITY OF OREGON OSAKA UNIVERSITY

\section{UNIVERSITY OF SOUTHERN CALIFORNIA STANFORD UNIVERSITY UNIVERSITY OF TOKYO UNIVERSITY OF UTAH WASHINGTON STATE UNIVERSITY UNIVERSITY OF WASHINGTON AMERICAN MATHEMATICAL SOCIETY NAVAL WEAPONS CENTER}

* C. R. DePrima California Institute of Technology, Pasadena, CA 91109, will replace J. Dugundji until August 1974. 


\section{Pacific Journal of Mathematics}

\section{Vol. 49, No. $1 \quad$ May, 1973}

A. Bigard, Free lattice-ordered modules ...........................

Richard Bolstein and Warren R. Wogen, Subnormal operators in strictly cyclic

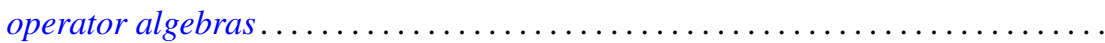

Herbert Busemann and Donald E. Glassco, II, Irreducible sums of simple

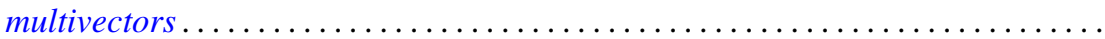

W. Wistar (William) Comfort and Victor Harold Saks, Countably compact groups

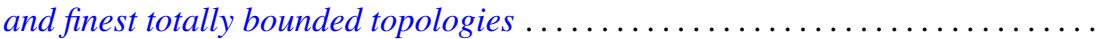

Mary Rodriguez Embry, Maximal invariant subspaces of strictly cyclic operator

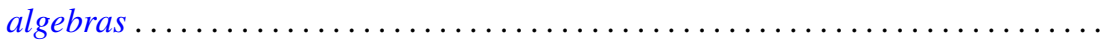

Ralph S. Freese and James Bryant Nation, Congruence lattices of semilattices......

Ervin Fried and George Grätzer, A nonassociative extension of the class of

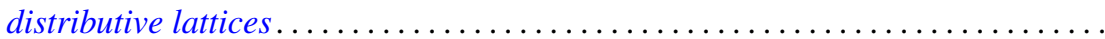

John R. Giles and Donald Otto Koehler, On numerical ranges of elements of locally

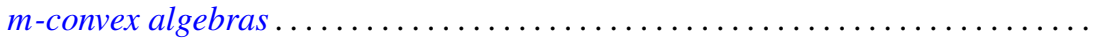

David A. Hill, On dominant and codominant dimension of $\mathrm{QF}-3$ rings ........ John Sollion Hsia and Robert Paul Johnson, Round and Pfister forms over $R(t) \ldots$ I. Martin (Irving) Isaacs, Equally partitioned groups . . . . . . . . . . . . . .

Athanassios G. Kartsatos and Edward Barry Saff, Hyperpolynomial approximation of solutions of nonlinear integro-differential equations.

Shin'ichi Kinoshita, On elementary ideals of $\theta$-curves in the 3-sphere and 2-links in the 4-sphere

Ronald Brian Kirk, Convergence of Baire measures

R. J. Knill, The Seifert and Van Kampen theorem via regular covering spaces ..

Amos A. Kovacs, Homomorphisms of matrix rings into matrix rings ..

Young K. Kwon, HD-minimal but no $H D$-minimal ..........

Makoto Maejima, On the renewal function when some of the mean renewal lifetimes are infinite

Juan José Martínez, Cohomological dimension of discrete modules over profinite groups.

W. K. Nicholson, Semiperfect rings with abelian group of units

Louis Jackson Ratliff, Jr., Three theorems on imbedded prime divisors of principal ideals.

Billy E. Rhoades and Albert Wilansky, Some commutants in $B(c)$ which are almost matrices

John Philip Riley Jr., Cross-sections of decompositions . . .

Keith Duncan Stroyan, A characterization of the Mackey uniformity $m\left(L^{\infty}, L^{1}\right)$ for

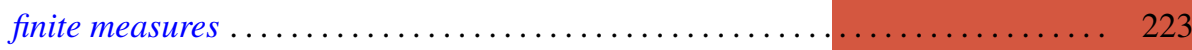

Edward G. Thurber, The Scholz-Brauer problem on addition chains . . . . . . . . . 229

Joze Vrabec, Submanifolds of acyclic 3-manifolds ............

Philip William Walker, Adjoint boundary value problems for compactified singular

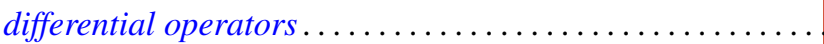

